Rez. zu Logi GUNNARSSON, Vernunft und Temperament. Eine Philosophie der Philosophie

Paderborn, Mentis-Verlag, 2020

\title{
Magnus Schlette
}

\section{(2) OpenEdition}

\section{Journals}

Electronic version

URL: http://journals.openedition.org/ejpap/2358

DOI: 10.4000/ejpap.2358

ISSN: 2036-4091

Publisher

Associazione Pragma

\section{Electronic reference}

Magnus Schlette, "Rez. zu Logi gunnarsson, Vernunft und Temperament. Eine Philosophie der Philosophie", European Journal of Pragmatism and American Philosophy [Online], XIII-1 | 2021, Online since 02 April 2021, connection on 04 April 2021. URL: http://journals.openedition.org/ejpap/2358 ; DOI: https:// doi.org/10.4000/ejpap.2358

This text was automatically generated on 4 avril 2021.

\section{$\Theta \oplus \Theta \Theta$}

Author retains copyright and grants the European Journal of Pragmatism and American Philosophy right of first publication with the work simultaneously licensed under a Creative Commons AttributionNonCommercial-NoDerivatives 4.0 International License. 


\section{Rez. zu Logi GUNNARSSON, Vernunft und Temperament. Eine Philosophie der Philosophie}

Paderborn, Mentis-Verlag, 2020

Magnus Schlette

\section{REFERENCES}

Logi GUNNARSSON, Vernunft und Temperament. Eine Philosophie der Philosophie, Paderborn, Mentis-Verlag, 2020, 447 S.

\section{In den Spuren Kierkegaards. Logi Gunnarsson philosophiert via William James über den Sinn des Philosophierens}

1 In einer Tagebuchaufzeichnung Sören Kierkegaards aus den 1830'er Jahren erfahren wir von der Suche des Verfassers nach einer Wahrheit, die "Wahrheit für mich ist" (Kierkegaard 1962: 16), und von seiner Einsicht, dies sei es gewesen, was ihm gefehlt habe: "ein vollkommen menschliches Leben $\mathrm{zu}$ führen, und nicht bloß eins der Erkenntnis, um dadurch so weit zu kommen, daß ich meine Gedankenentwicklungen nicht gründete auf [...] etwas, was doch auf jeden Fall nicht mein eigen ist, sondern auf etwas, was mit der tiefsten Wurzel meines Daseins zusammenhängt [...]" (ebd.: 17f). Diese Zeilen sind oftmals als charakteristisch für die existenzphilosophische Denkungsart gedeutet worden, deren geistesgeschichtlicher "Durchbruch" mit dem Namen Kierkegaards verbunden wird; für die Entfremdung der Philosophen von der Selbstgenügsamkeit akademischer Fachdiskurse, die in hochmütiger Distanz zu jeglichen praktischen Belangen über theoretische Geltungsansprüche befinden, und für ihre Hinwendung zu einer Rechenschaftsgabe über die Bedeutung ihres Denkens für die 
Lebensführung. Kierkegaard wirft darüber hinaus die metaphilosophische Frage auf, ob nicht auch der Stil des Philosophierens und die Form philosophischer Werke sich ändern müssten, wenn sie der Einsicht in die intrinsische Verknüpfung zumindest einiger zentraler theoretischer Wahrheitsprobleme mit der Rolle, die sie im Leben spielen, gerecht werden sollen. Die Antwort, die Kierkegaard auf diese Frage mit einer bis dato unerhörten Konsequenz erprobt hat, besteht darin, dass er seine Philosopheme von fiktiven personae vortragen lässt, die er in kunstvollen literarischen Rahmenhandlungen einführt und zueinander in Beziehung setzt: Den Leserinnen und Lesern werden nicht einfach Behauptungen und Begründungen aufgetischt, sondern diese spiegeln immer auch die Art und Weise wieder, wie sie von denjenigen Figuren, die Kierkegaard sie aufstellen lässt, angeeignet und zu einem integralen Bestandteil ihrer praktischen Selbst- und Weltverständigung gemacht worden sind.

2 Logi Gunnarsson wandelt in seinem neuen Buch Vernunft und Temperament. Eine Philosophie der Philosophie in vielschichtiger und ganz eigenständiger Weise auf den Spuren Kierkegaards. Es geht darin um die Explikation der grundlegenden Intuition, dass ein guter Philosoph ein wahrer Mensch sein müsse (Gunnarsson 2020: 19). Oder geht der Rezensent mit dieser Behauptung in ähnlicher Weise dem Verfasser auf den Leim wie der Interpret von Entweder/Oder Sören Kierkegaard, wenn er diesem das Persönlichkeitskonzept des fiktiven Gerichtsrats Wilhelm zuschreibt? Denn wie Kierkegaard sich aus Entweder/Oder zugunsten seiner Kunstfiguren herausgehalten hat, so zieht sich auch Gunnarsson hinter zwei fiktive Philosophen zurück, die gemeinsam und im Widerstreit miteinander ein Buchmanuskript verfasst haben. Was immer wir in Vernunft und Temperament lesen, müssen wir entweder dem einen oder dem anderen der beiden zuschreiben - als da sind: Bill Headstrong, ehrgeiziger und selbstbewusster amerikanischer Assistant Professor, sportiv, drahtig und blitzgescheit, der die Publikation des geplanten Buches im Anlauf auf eine Tenure Track-Position benötigt, und Wilhelm Kornblum, ein eher empfindsam veranlagter, bereits älterer, daher auch gelassenerer und lebenserfahrenerer Philosoph, "väterlicher Freund" von Headstrong wie Kierkegaards Gerichtsrat vom jungen Ästhetiker in Entweder/Oder. Headstrong und Kornblum planen nach anfänglich entdeckter "Seelenverwandtschaft," wie es Kornblum in einem Brief an den Jüngeren formuliert, ein Buch zu schreiben, das im Ausgang von einer Auseinandersetzung mit ihrem gemeinsamen Helden William James Sinn und Aufgabe der Philosophie erkunden soll, mit anderen Worten: eine Philosophie der Philosophie zu schreiben, wie sie der Untertitel von Vernunft und Temperament ankündigt. Dass ein guter Mensch ein wahrer Mensch sein müsse, versteht Kornblum so, dass philosophische Fragen und die Beschäftigung mit der eigenen Lebensführung sich gegenseitig durchdringen müssten (Gunnarsson 2020: 127). Er und Headstrong sind sich einig, dass es philosophische Theorien gebe, über deren Wahrheitsgehalt nicht einfach theoretisch entschieden werden könne, weil er intrinsisch mit der Rolle verknüpft sei, die diese Theorien im Leben spielen. Beide bemühen sich auf ganz unterschiedliche und - wie sich im Verlauf des Buches zeigt - unvereinbare Weise, diese ihnen beiden gemeinsame Grundüberzeugung zu entwickeln.

3 Ausgangspunkt ihrer gemeinschaftlichen Erörterung ist die Lebenskrise, die William James um das Jahr 1870 herum durchgemacht hat und zu deren Veranschaulichung die beiden sich vor allem auf zwei Tagebucheinträge von James vom 30. April 1870 und vom 10. April 1873 berufen. Die Krise war ausgelöst worden durch James' Auseinandersetzung mit dem Naturalismus. Insbesondere die Infragestellung der 
objektiven Gültigkeit moralischer Forderungen und der menschlichen Willensfreiheit konfrontiert James mit der Möglichkeit eines sinnlosen Universums. James verzweifelt nicht etwa am naturalistischen Weltbild als solchem, sondern an der Pattsituation, die er dem Streit zwischen den Theorien des moralischen Objektivismus und des moralischen Subjektivismus, der Willensfreiheit und des Determinismus attestiert. In dieser Situation bezeugt der ältere Tagebucheintrag James' Entscheidung, angesichts des Stellungskriegs zwischen den Weltbildern an den freien Willen zu glauben. Drei Jahre später notiert James in seinem Tagebuch, es sei gar nicht notwendig, "die universellen Probleme direkt und als solche in ihrer abstrakten Form anzugehen" (James 1873; zit. n. Gunnarsson 2020: 101). Kornblum und Headstrong interpretieren die beiden Einträge als Etappen auf dem Weg der Herausbildung der philosophischen Einsicht, dass über die Wahrheit dieser philosophischen Theorien nicht allein auf theoretischem Wege entschieden werden könne. Beide sind der Meinung, dass James eine Neukonzeption der Philosophie anpeilt, für die er in der Kreativphase seiner philosophischen Aufsätze um das Jahr 1880 herum die entscheidenden Weichen gestellt habe. Diese Einsicht beschränke sich keineswegs darauf, wie der erste der erwähnten Tagebucheinträge insinuiert, aus der Pattsituation zwischen philosophischen Theorien mit gleichwertiger Kohärenz die Berechtigung abzuleiten, der jeweils lebensförderlicheren den Glauben zu schenken. Die entscheidende Pointe von James, die er um 1880 herum ausarbeite, bestehe nicht in der besagten Glaubensberechtigung, sondern in einer neuen Form von Wahrheitsbegründung, die der epistemischen Validität nicht-theoretischer Gründe Rechnung trage.

Headstrong argumentiert in den von ihm verantworteten Buchpartien dafür, James habe die Position des internen Objektivismus ausgearbeitet, derzufolge die Gründe, die philosophische Wahrheiten konstitutiv erklären, und menschliche Empfindungen begrifflich voneinander abhängig seien. James postuliere ein Passungsverhältnis zwischen unseren Konzepten von der Beschaffenheit der Welt und unseren Konzepten von den jeweils stimmigen Reaktionsweisen darauf, wie die Welt und das menschliche Weltverhältnis beschaffen sind. Für das Problem der Willensfreiheit heißt das, dass sich nur durch die begriffliche Angemessenheit, mit der wir unsere Reaktion auf ein (Fehl-)Verhalten als Bedauern prädizieren oder eine Verantwortungszuschreibung als fair bewerten, konstitutiv erklären können, "warum es echte Möglichkeiten in der Welt gibt" (Gunnarsson 2020: 284), warum also ein inkompatibilistisches Freiheitsverständnis begrifflich gerechtfertigt ist. Und Headstrong knüpft an James' philosophische Einsicht an, um sie im Kontext der Gegenwartsphilosophie zu einer allgemeinen Theorie philosophischer Gründe, einer Art road map auf dem Wege der Beantwortung von Fragen wie denjenigen auszuarbeiten, was eine philosophische Theorie der Willensfreiheit wahr macht (ebd.: 357ff) und welche Gründe entscheiden, ob die Moral eine Projektion ist (366ff). Kornblum spürt wiederum den Konsequenzen nach, die sich daraus für die Relevanz der Lebenserfahrung bei der Ergründung des Wahrheitswerts philosophischer Theorien über Themen wie die Willensfreiheit oder die moralische Richtigkeit ergeben. Man müsse diese Theorien eben im Leben testen: Es gehöre notwendigerweise zu den philosophischen Begriffen, dass die philosophische Theorie stets nur eine unvollständige Interpretation dieser Begriffe sein könne. "Die Bestätigung der Theorie in der Praxis ist also nicht bloß die Bestätigung von etwas Allgemeinen, sondern liefert auch selbst zugleich ein ständig komplexeres Verständnis des Begriffs selbst" (406). 
5 Als Kornblum diesen Gedanken festhält, haben sich seine Wege und die seines jungen amerikanischen Kollegen aber schon wieder getrennt. Denn die beiden haben sich niemals darauf einigen können, welche sprachliche Form ihrer Philosophie der Philosophie, die sie aus der Auseinandersetzung mit James' entwickeln wollten, tatsächlich angemessen sei. Und dieser Streit betraf sowohl die philologischhermeneutische Auseinandersetzung mit den Quellen, also mit James' Philosophie und seinen Lebenszeugnissen, wie die systematisch-philosophischen Konsequenzen, die sie daraus ziehen wollten. Vernunft und Temperament ist ein Fragment, in dem einzelne Buchkapitel der beiden Autoren Kornblum und Headstrong einander folgen, miteinander um den rechten Begriff und die rechte Form philosophischer Rechenschaftsgabe über das philosophische Wahrheitsstreben konkurrieren, einander herausfordern, relativieren und widersprechen. Die Kapitelfolge ist durchsetzt mit dem Briefwechsel der beiden Helden, die sich voreinander für ihre so unterschiedliche Herangehensweise an das gemeinsame Projekt rechtfertigen, die einander kritisieren und wechselseitig in Frage stellen.

6 Für Kornblum folgt aus der intrinsischen lebenspraktischen Ergänzungsbedürftigkeit philosophischer Theorien, dass die Interpreten des Werks von James wissen müssen, "wie er sich als Mensch mit den philosophischen Lebensfragen beschäftigt hat" (32). Philosophische Einsichten seien so beschaffen, dass sie "mit ihrem verwandelnden Bedeutungsgewicht in alle Dimensionen des Lebens" eindringen, so Kornblum mit Dieter Henrich (Henrich 2011: 27; zit. n. Gunnarsson 2020: 74). Dem müsse auch eine Auseinandersetzung mit James Rechnung tragen. Kornblum bewegt sich hier nebenbei auf den Pfaden Georg Simmels, der auf der Grundlage derselben Prämissen, die Kornblum von der Lebensbedeutsamkeit philosophischer Einsichten vertritt, bereits um 1900 schrieb, dass die tiefere Aufgabe der genuin philosophischen Deutung philosophischer Werke "statt auf die bloße Darlegung der Philosophie des Denkers auf eine Philosophie über den Denker gehen muß" (Simmel 1995: 169). Ist wiederum der philosophische Gedanke, so Simmel, "das sublimierteste Resultat eines weitausgreifenden Lebens, das uns jener Gedanke nachzuerleben, bis es in ihn selbst mündet, veranlaßt" (Simmel 1996: 11), dann folgt daraus, dass die philosophische Werkdeutung nicht von der Lebensgeschichte abstrahieren darf, in der sich die philosophische Einsicht herausgebildet hat. So ist denn auch für Kornblum die Biographik Teil der philosophischen Deutung genuin philosophischer Einsichten. Die sprachliche Form, die der intrinsischen Verbindung von Denken und Leben Rechnung trage, sei die philosophische Erzählung: "Sie muss einen Protagonisten haben, der über das Leben, das er gerade führt, philosophiert. Seine philosophische Haltung muss auf sein Leben antworten und sein Leben muss von seiner philosophischen Haltung durchdrungen sein" (Gunnarsson 2020: 84). Konsequenterweise rekonstruiert er den systematischen Gehalt von James' philosophischer Einsicht aus den Jahren seiner Lebenskrise in einem der Buchkapitel in Gestalt eines philosophischen Tagebuchs. Das wiederum ruft den Protest von Headstrong auf den Plan. Kornblum vermenge seine Überlegungen mit James' Ansatz, ohne darauf hinzuweisen, und nehme sich die Freiheit, den fiktiven Autor seines philosophischen Tagebuchs Auffassungen vertreten $\mathrm{zu}$ lassen, für die es keinen Hinweis gebe, dass James sie jemals vertreten habe (ebd.: 220). Headstrong scheint nicht zu erkennen, dass Kornblums Verflechtung von Objektund Metasprache Methode hat und sich einem grundsätzlich anderen Philosophieverständnis verdankt als seinem eigenen. Kornblums Antwort auf Headstrong steht der Sache nach in der Tradition Simmels: Er habe sein 
philosophisches Tagebuch als eine Einladung an den Leser verstanden, "selbst eine solche Reise wie der Autor des Textes zu unternehmen" (223). Davon will Headstrong wiederum nichts wissen: So sehr theoretische Gründe intrinsisch mit Emotionen und Lebenserfahrungen verknüpft sein mögen, müsse diese Einsicht gleichwohl theoretisch, auf dem Wege einer analytisch stringenten Argumentation herausgearbeitet werden.

In seinem Abschiedsbrief, mit dem Kornblum die gemeinsame Arbeit aufkündigt, spitzt sich der Dissens bis zu dem für Philosophen wohlmöglich schwersten Vorwurf mangelnder intellektueller Redlichkeit an den Jüngeren zu: "Du behauptest, These $\mathrm{X}$ könne nur im Leben auf ihre Wahrheit getestet werden. Zugleich vertrittst Du These X, ohne sie im Leben getestet $\mathrm{zu}$ haben. Das muss ich leider, lieber Bill, Verlogenheit nennen" (382). Aber was folgt daraus? Für Kornblum der Abschied von der akademischen Philosophie: Er kündigt seine Professur, um an anderem Ort unter anderem Namen ein neues Leben, ein philosophisches Leben zu beginnen, das Raum dafür bietet, praktisch zu testen, was sich theoretisch nicht erweisen lässt. Und Headstrong? Er veröffentlicht das Fragment ihrer Zusammenarbeit in der Hoffnung, es werde vor dem Tenure Track-Komitee Gnade finden. Und Gunnarsson? Wie Kierkegaard die ihn bewegenden Probleme hält er auch die seinen in der Schwebe. Weder muss er noch im Laufrad für die Entfristung seiner Professur strampeln, noch hat er sie (bislang) aufgegeben. Er legt sich auch nicht auf einen der intellektuellen Persönlichkeitstypen fest, die uns in Gestalt Kornblums und Headstrongs begegnen. Weder lässt er sich zu billiger Institutionenschelte herab noch schwingt er sich zur Romantisierung eines philosophischen Vagabundenlebens auf. Er geht aber auch nicht den umgekehrten Weg. Die Pointe seiner Philosophie der Philosophie ist, dass es die eine Philosophie der Philosophie nicht gibt. Und auch nicht die eine Art und Weise der philosophischen Deutung philosophischer Werke.

8 Sein Buch ist aber auch kein Votum für einen Relativismus des "Anything goes." Im Handbuch philosophischer Grundbegriffe verrät uns der Eintrag unter dem Stichwort Philosophie: "Der Begriff der Philosophie ist selbst Gegenstand der Philosophie. In diesem Sachverhalt sind alle jene Aporien zusammengefasst, die sich bei dem Versuch ergeben, einen Begriff der Philosophie zu fixieren. [...] Die Philosophie ist schon am Werk und mithin schon bestimmt, wenn sie daran geht, sich selbst zu bestimmen" (Krings u.a. 1973). Das Handbuch verrät uns freilich nicht, warum das so ist. Gunnarssons Interpretation der philosophischen Einsicht von James in die begriffliche Abhängigkeit des Wahrheitswerts philosophischer Theorien von emotionsbezogenen Gründen ist eine plausible Antwort darauf. Seine Charakterisierung völlig unterschiedlicher philosophischer Persönlichkeiten, durch die er diese Interpretation vortragen lässt, eine andere (wiewohl beide Antworten recht besehen miteinander zu tun haben). Außerdem zeigt Gunnarsson, dass das, was laut dem zitierten Handbuch für die Philosophie gilt, auch auf die Philosophie der Philosophie zutrifft. Er legt eine äußert bemerkens- und bedenkenswerte Interpretation der jamesianischen Einsicht vor, eine Interpretation, die sich in der Konsequenz, mit der sie das Formproblem der Philosophie behandelt, in den Spuren Kierkegaards bewegt, aber in einer frischen und aktuellen Weise, die gegenwartsphilosophisch up to date ist. Sein Buch gehört zu den wenigen, die die geneigten Leserinnen und Leser ganz und ruhig auch zweimal lesen sollten, denn wem von beiden, Kornblum oder Headstrong, ihre Gunst gilt, will wohl überlegt sein. Am Ende ist auch dies eine Frage von empfindungsbezogenen Gründen. 


\section{BIBLIOGRAPHY}

HENRICH Dieter, (2011), Werke im Werden. Über die Genesis philosophischer Einsichten, München, C. H. Beck Verlag.

JAMES William, (1873), William James papers, Houghton Library, Harvard University, bMS Am 1092.9 (4550), Eintrag vom 10. April 1873.

KIERKEGAARD Sören, (1962), Die Tagebücher. Erster Band, hg. u. übers. v. Hayo Gerdes, Düsseldorf/ Köln, Eugen Diederichs Verlag.

KRINGS Hermann u.a. (Hgg.), (1973), Handbuch philosophischer Grundbegriffe, Band 2, München, Kosel.

SIMMEL Georg, (1995), "Schopenhauer und Nietzsche," in ders. Gesamtausgabe, Band 10, Frankfurt am Main, Suhrkamp.

SIMmel Georg, (1996), "Hauptprobleme der Philosophie," in ders. Gesamtausgabe, Band 14, Frankfurt am Main, Suhrkamp.

\section{AUTHORS}

MAGNUS SCHLETTE

Universität Heidelberg

magnus.schlette[at]fest-heidelberg.de 